\title{
EXPONENTIAL STABILITY AND TRANSFER FUNCTIONS OF PROCESSES GOVERNED BY SYMMETRIC HYPERBOLIC SYSTEMS
}

\author{
Cheng-Zhong Xu ${ }^{1}$ and Gauthier Sallet ${ }^{1}$
}

\begin{abstract}
In this paper we study the frequency and time domain behaviour of a heat exchanger network system. The system is governed by hyperbolic partial differential equations. Both the control operator and the observation operator are unbounded but admissible. Using the theory of symmetric hyperbolic systems, we prove exponential stability of the underlying semigroup for the heat exchanger network. Applying the recent theory of well-posed infinite-dimensional linear systems, we prove that the system is regular and derive various properties of its transfer functions, which are potentially useful for controller design. Our results remain valid for a wide class of processes governed by symmetric hyperbolic systems.
\end{abstract}

Mathematics Subject Classification. 93D09, 93D25, 80A20, 35L50.

Received August 6, 2001. Revised January 23, 2002.

\section{INTRODUCTION}

In all chemical engineering processes which have heat, mass or momentum transfer, there exist gradients in spatial directions. Some processes are specifically designed to take advantage of gradients along the axis of flow. The tubular reactor, the shell and tube heat exchangers and the packed mass exchange columns all achieve their objectives in this way [9]. The dynamics of most of these processes must be described by partial differential equations (PDE's), resulting in distributed parameter systems. In this paper we study frequency and time domain behaviour of a class of hyperbolic systems represented by a countercurrent heat exchanger network. This class of systems covers classical countercurrent heat exchangers [9], packed mass exchange columns [9,38] and irrigation canals $[2,5,40]$. Our approach is essentially infinite-dimensional: the analysis will be carried out based on PDE models, in contrast to the discrete approximation method, which results in finite-dimensional models ( $c f$. Hangos et al. [11]).

The network of countercurrent heat exchangers to be considered is governed by two sets of hyperbolic partial differential equations:

$$
\left\{\begin{array}{l}
\frac{\partial R_{1}}{\partial t}=m_{1} \frac{\partial R_{1}}{\partial x}-K_{1}\left(R_{1}-R_{2}\right)+b_{1}(x) u(t) \\
\frac{\partial R_{2}}{\partial t}=-m_{2} \frac{\partial R_{2}}{\partial x}+K_{2}\left(R_{1}-R_{2}\right)
\end{array}\right.
$$

\footnotetext{
Keywords and phrases: Heat exchangers, symmetric hyperbolic equations, exponential stability, regular systems, transfer functions.

1 Inria (CORIDA, CONGE) \& ESA 7035 du CNRS (MMAS), bâtiment A, Université de Metz, 57045 Metz Cedex 01, France; e-mail: $\mathrm{xu}$, sallet@loria.fr
} 
on the domain $(x, t) \in\left(0, l_{1}\right) \times \mathbb{R}^{+}$(where $\left.\mathbb{R}^{+}=(0,+\infty)\right)$, and

$$
\left\{\begin{array}{l}
\frac{\partial T_{1}}{\partial t}=(1-\beta) m_{1} \frac{\partial T_{1}}{\partial x}-K_{1}\left(T_{1}-T_{2}\right)+b_{2}(x) u(t) \\
\frac{\partial T_{2}}{\partial t}=-m_{2} \frac{\partial T_{2}}{\partial x}+K_{2}\left(T_{1}-T_{2}\right)
\end{array}\right.
$$

on the domain $(x, t) \in\left(l_{1}, l_{2}\right) \times \mathbb{R}^{+}$. (For the sake of simplicity we write $R_{i}$ for $R_{i}(x, t), i=1,2$ and similarly for $T_{i}$.) These PDE's are coupled by the boundary conditions

$$
\left\{\begin{array}{l}
R_{1}\left(l_{1}, t\right)=(1-\beta) T_{1}\left(l_{1}, t\right) \\
R_{2}\left(l_{1}, t\right)=T_{2}\left(l_{1}, t\right) \\
T_{1}\left(l_{2}, t\right)=0 \\
R_{2}(0, t)=d(t) .
\end{array}\right.
$$

We consider the following output:

$$
y(t)=T_{2}\left(l_{2}, t\right) .
$$

In the above equations $m_{i}, K_{i}, l_{i}$ with $i=1,2$ and $\beta$ are positive constants such that $l_{2}>l_{1}>0$ and $0<\beta<1$. The state variables $R_{i}(x, t)$ and $T_{i}(x, t), i=1,2$ are deviations of the temperatures of fluids from their steady state values. (The reader is referred to Gauthier and $\mathrm{Xu}[10]$ for a detailed list of variables in the equations together with their physical meaning.) This heat exchanger model has been constructed in [10] in order to simulate the qualitative input-output behaviour of an industrial furnace. It consists of two countercurrent heat exchangers connected in cascade. When we consider fluid flow rates as control variables, the resulting control system is bilinear. Linearizing the bilinear system around a steady state point we get the linear system described above. Hence the functions $b_{1}(x)$ and $b_{2}(x)$ are continuously differentiable and determined uniquely by the steady state value (see [10]). The control variable $u(t)$ represents the variation of the fluid flow rate. The disturbance variable $d(t)$, representing the variation of the fluid inlet temperature, enters into the boundary condition (1.3). Thus the equations (1.1-1.4) describe an infinite-dimensional linear system with boundary and distributed input and boundary output. As shown in [10], the system is non-minimum phase: the transfer function from $u$ to $y$ has zeros in the open right half-plane. The $H^{\infty}$ control theory developed in [8] has been applied in [10] to this system for minimizing the worst effect of the disturbance $d(t)$ on the output $y(t)$. The industrial interest of the resulting $H^{\infty}$ controller has been explained in [10].

In this paper we show how the system (1.1-1.3) is transformed into a dissipative symmetric hyperbolic system. Then we prove that the associated semigroup is exponentially stable using a theorem of Rauch and Taylor [25]. We prove that the system (1.1-1.4) is regular in the sense of Weiss [32]. The regularity of the controlled and observed system with exponential stability of the semigroup guarantees that the transfer functions $P(s)$ and $W(s)$ (corresponding to the input-output mappings $u \rightarrow y$ and $d \rightarrow y$, respectively) are in $H^{\infty}$ (that is, analytic and bounded on the open right half-plane). This gives a positive answer to some of the open questions in [10]. The fact that the system under consideration is regular has useful consequences on the design of feedback controllers for the system. The cascade connection of two regular systems is again regular. The class of regular linear systems is closed under feedback (see [31]). The most important consequence is that internal stability and external stability are equivalent for a regular system which is both stabilizable and detectable, as proved in Rebarber [26] (see also Morris [19], Weiss and Curtain [33]).

As pointed out in Ydstie and Alonso [42], many thermodynamic processes satisfying a system of conservation laws are governed in particular by symmetric hyperbolic systems of PDE's. We propose studying the problem of stability and well-posedness for this class of systems based on the heat exchanger network. We propose a new proof of the theorem of Rauch and Taylor under an additional assumption by using the direct method of Lyapunov. The advantage of this method is its simplicity and the fact that it is not limited to one space variable. 
The direct method of Lyapunov allows us to attack symmetric hyperbolic systems in higher dimensions and degenerate hyperbolic cases that the theory in [25] cannot treat, see our Examples in Section 2.

Countercurrent heat exchanger systems are usually supposed to be stable by process engineers without mathematical proof. From [42], for a strictly dissipative process with the action satisfying the Clausius-Planck property the state variable tends to a passive state as time goes to infinity. In particular, a countercurrent heat exchanger is a dissipative process. However the infinite-dimensional feature of the systems makes it necessary to investigate if the stability is structural and exponential. The exponential stability of the classical heat exchangers has been proved in [35] by the direct method of Lyapunov when the diffusion term is taken into account in the flow. For the hyperbolic case (without diffusion) only strong stability has been obtained in [35] by the decomposition theorem of [1]. In [36] we have established exponential stability for the hyperbolic case using Huang's theorem [13] (or Prüss's theorem [23]). Here, the application of the theory of symmetric hyperbolic systems makes it possible to prove exponential stability for a much wider class of processes. In particular, networks of heat exchangers formed by cascade connection are in this class. Many processes such as tubular reactors, gas absorbers and irrigation canals can be modeled as symmetric hyperbolic systems (see $[2,5,9,27,39,40]$ and [35]). Using the recent representation theory as developed in Weiss [32] we are able to characterize the transfer functions in terms of the semigroup operator, control operator and observation operator. Moreover we find the inner-outer factorization for some of them. This characterization is useful for various controller designs, see Logemann et al. [17], Logemann and Townley [18], Staffans [29] and Weiss and Curtain [33]. While the class of PDE models that we investigate is related to process systems with one spatial coordinate, no diffusion (only convective transport) and no source (only heat exchange), our theoretical results can be extended to deal with nonlinearities and energy transformations that take place in a reactive system (cf. Xu and Feng [34,41]). For more aspects of nonlinear hyperbolic systems the reader is referred to Coron et al. [5] and $\mathrm{Li}$ [16] as well as the references there.

Since the work [25] of Rauch and Taylor in 1974 important developments have been achieved for stability in the field of symmetric hyperbolic systems on $\mathbb{R}^{d}$ ( $c f$. Kreiss et al. [14], Oritiz [20] and the references there). We should mention the recent research carried out by Kreiss et al. [14] and Oritiz [20] in this direction. In Kreiss et al. [14] asymptotic stability has been studied for solutions which are periodic w.r.t. space variables or equivalently with bounded open cubic domain subject to periodic boundary conditions. It has been shown that some eigenvalue condition for the symbols of the first order partial differential operator implies exponential stability of the corresponding symmetric hyperbolic system. Roughly speaking, after the Fourier series transform w.r.t. the space variables (the PDE becoming ODE w.r.t. time) they have exploited the Lyapunov method in the frequency domain in order to establish the stability result. The stability problem that we consider is concerned with initial-boundary symmetric hyperbolic systems. We have both initial condition and boundary condition to be satisfied. If we assume the so-called eigenvalue condition in our case exponential stability is somehow trivial. In [20], motivated by physical systems describing dissipative relativistic fluids Ortiz has considered the stability problem for symmetric hyperbolic PDE on the whole Euclidean space $\mathbb{R}^{d}$. It has been proved in Ortiz [20] that if $d \geq 3$ some similar eigenvalue condition on the constant coefficients system symbol implies the decay of the solution to zero as time goes to infinity. The result has been established using the Laplace transform w.r.t. time and the Fourier transform w.r.t. space. Once more the condition is too restrictive for our initial-boundary case. Our approach for stability is different: we exploit the Lyapunov method in the time domain. Our result is concerned with boundary dissipations. When the boundary is really dissipative we expect exponential stability. As the same as in Kreiss et al. [14] and in Ortiz [20] our stability result remains valid for small nonlinear perturbations. The contribution of our paper is proposing appropriate Lyapunov functionals for some initial-boundary symmetric hyperbolic systems and proving the well-posedness of these systems with control and observation. The result that we obtain in the time domain gives us informations on the frequency domain.

Our paper is organized as follows. In Section 2 the stability result of Rauch and Taylor is presented for dissipative symmetric hyperbolic systems. We propose an alternative proof using Lyapunov functionals, under an additional symmetry assumption. In Section 3 we transform the equations (1.1-1.3) into a symmetric 
hyperbolic system. Exponential stability is established for the system by applying the result presented in Section 2. We prove that the system, with distributed and/or boundary control and boundary observation, is well-posed. In Section 4 we prove further that the system is regular and give explicit expression for its transfer functions and the associated feedthrough operators. The inner-outer factorization and various properties are deduced for some of them. Section 4 contains conclusions.

\section{Symmetric HyPERBolic Systems Without CONTROL}

In the first part of this section we present our general idea for the proof of the Rauch and Taylor theorem. In the second part we illustrate possible generalizations of our idea via two examples. The first example is concerned with degenerate hyperbolic systems and the second one is concerned with hyperbolic systems of more than two independent variables.

Consider a symmetric hyperbolic system of the form:

$$
\left\{\begin{array}{l}
\frac{\partial R(x, t)}{\partial t}=A(x) \frac{\partial R(x, t)}{\partial x}+B(x) R(x, t), \quad(x, t) \in(0,1) \times \mathbb{R}^{+} \\
R^{-}(0, t)=D_{0} R^{+}(0, t) \\
R^{+}(1, t)=D_{1} R^{-}(1, t) \\
R(x, 0)=R_{0}(x)
\end{array}\right.
$$

where $R(x, t)$ is an $n \times 1$ vector function for $(x, t) \in(0,1) \times \mathbb{R}^{+}$, partitioned as

$$
R(x, t)=\left(\begin{array}{c}
R^{-}(x, t) \\
R^{+}(x, t)
\end{array}\right)
$$

with $R^{-}(x, t) \in \mathbb{R}^{p}$ and $R^{+}(x, t) \in \mathbb{R}^{q}$, respectively and $p+q=n . B(x)$ is a real $n \times n$ matrix function and $A(x)$ is a diagonal matrix function for $x \in[0,1]$, and $D_{0}$ and $D_{1}$ are real constant matrices. The diagonal matrix $A(x)$ is partitioned as

$$
A(x)=\left(\begin{array}{lr}
A^{-}(x) & 0 \\
0 & A^{+}(x)
\end{array}\right)
$$

with

$$
A^{-}(x)=\operatorname{diag}\left(\lambda_{1}(x), \lambda_{2}(x), \ldots, \lambda_{p}(x)\right)
$$

and

$$
A^{+}(x)=\operatorname{diag}\left(\lambda_{p+1}(x), \lambda_{p+2}(x), \ldots, \lambda_{p+q}(x)\right) .
$$

We denote by $\Lambda^{*}$ the transposed matrix of $\Lambda$ or the adjoint operator of $\Lambda$, as will be clear from the context, and by $A_{x}(x)$ the derivative of $A(x)$. We denote by $I$ the identity operator on any space. We assume that the following hypotheses are satisfied:

H.1) $B(\cdot) \in C^{0}\left([0,1] ; \mathbb{R}^{n \times n}\right)$ and $A(\cdot) \in C^{1}\left([0,1] ; \mathbb{R}^{n \times n}\right)$.

H.2) $\lambda_{i}(x)<0, i=1,2, \ldots, p$ and $\lambda_{p+i}(x)>0, i=1,2, \ldots, q$, for any $x \in[0,1]$.

H.3) For each $x \in[0,1]$,

$$
\begin{gathered}
B(x)+B^{*}(x)-A_{x}(x) \leq 0, \\
A^{-}(1)+D_{1}^{*} A^{+}(1) D_{1} \leq-r^{-} \cdot I
\end{gathered}
$$


and

$$
A^{+}(0)+D_{0}^{*} A^{-}(0) D_{0} \geq r^{+} \cdot I
$$

where $r^{-} \geq 0$ and $r^{+} \geq 0$ are constants such that $r^{-}+r^{+}>0$.

Let the state space of system (2.1) be the real Hilbert space $X=\left(L^{2}(0,1)\right)^{n}$ equipped with the inner product:

$$
\langle f, g\rangle_{X}=\int_{0}^{1} \sum_{k=1}^{n} f_{k}(x) g_{k}(x) \mathrm{d} x
$$

Theorem 1 (Rauch and Taylor [25]). Let H.1-H.3 be satisfied for the system (2.1). Then, for each $R_{0} \in X$ the system has a unique solution $R(\cdot, t) \in C([0,+\infty) ; X)$. Moreover the semigroup of bounded linear operators $U(t)$ on $X$ such that $R(\cdot, t)=U(t) R_{0}$ is exponentially stable:

$$
\|U(t)\|_{\mathcal{L}(X)} \leq M \mathrm{e}^{-\omega t}
$$

for some constants $M, \omega>0$.

Remark 1. The equations (2.1) are hyperbolic with two independent variables, and the proof in [25] uses the method of characteristics. Here, we propose an alternative proof for a subclass of the systems in (2.1) where $B(x)$ is symmetric: $B^{*}(x)=B(x)$ for all $x \in[0,1]$, using a Lyapunov function. The advantage of the direct method of Lyapunov is its simplicity and its potential usefulness for hyperbolic systems in more than one space variable. We demonstrate this by proving exponential stability of a symmetric hyperbolic system defined on an open bounded domain in $\mathbb{R}^{2}$.

Remark 2. For the exponential stability with $C^{1}$ topology on $X$ (instead of the $L^{2}$ topology), of nonlinear systems similar to $(2.1)$, the reader is referred to $\mathrm{Li}[16]$.

Proof of Theorem 1. Suppose that $B(x)$ is symmetric in (2.1). Let us consider the following candidate of Lyapunov function:

$$
V_{\theta}(R(\cdot, t))=\int_{0}^{1} R^{*}(x, t) \exp \left(\theta \int_{0}^{x} A(\xi) \mathrm{d} \xi\right) R(x, t) \mathrm{d} x, \quad \theta \in \mathbb{R} .
$$

For each $\theta>0, \sqrt{V_{\theta}(f)}$ induces on $X$ a norm of $f$ which is equivalent to the norm $\|f\|_{X}$ on $X$. From $(2.3)$ and (2.4) in H.3, we take $r^{-}>0$ without loss of generality. Define the unbounded operator $\mathcal{A}$ associated with the system (2.1) by

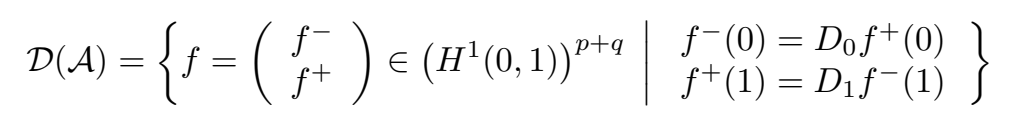

and for all $f \in \mathcal{D}(\mathcal{A})$,

$$
\mathcal{A} f(x)=A(x) \frac{\partial}{\partial x} f(x)+B(x) f(x)
$$

From [25] and [27] it is known that with H.1, H.2, $\mathcal{A}$ is the generator of a $C_{0}$-semigroup of bounded linear operators on $X$. Then $\mathbf{H . 3}$ implies that this semigroup is contractive. 
For each initial condition $R_{0} \in \mathcal{D}(\mathcal{A})$ we compute the derivative of $V_{\theta}(R(\cdot, t))$ along a trajectory of $(2.1)$ :

$$
\begin{aligned}
& \frac{\mathrm{d} V_{\theta}(R(\cdot, t))}{\mathrm{d} t}=-\theta \int_{0}^{1} R^{*}(x, t) A^{2}(x) \exp \left(\theta \int_{0}^{x} A(\xi) \mathrm{d} \xi\right) R(x, t) \mathrm{d} x \\
& +R^{*}(1, t) \exp \left(\theta \int_{0}^{1} A(\xi) \mathrm{d} \xi\right) A(1) R(1, t)-R^{*}(0, t) A(0) R(0, t) \\
& +\int_{0}^{1} R^{*}(x, t)\left(2 B(x)-A_{x}(x)\right) \exp \left(\theta \int_{0}^{x} A(\xi) \mathrm{d} \xi\right) R(x, t) \mathrm{d} x .
\end{aligned}
$$

From the second equation in (2.1) and (2.4), we obtain:

$$
R^{*}(0, t) A(0) R(0, t)=\left(R^{+}(0, t)\right)^{*}\left(A^{+}(0)+D_{0}^{*} A^{-}(0) D_{0}\right) R^{+}(0, t) \geq 0 .
$$

Each matrix being diagonal, using the second and third equations of (2.1) we can write

$$
\begin{aligned}
& R^{*}(1, t) \exp \left(\theta \int_{0}^{1} A(\xi) \mathrm{d} \xi\right) A(1) R(1, t)=\left\{\exp \left(\frac{\theta}{2} \int_{0}^{1} A^{-}(\xi) \mathrm{d} \xi\right) R^{-}(1, t)\right\}^{*} \\
& \left\{A^{-}(1)+D_{1}^{*} A^{+}(1) D_{1}+\left[\exp \left(-\frac{\theta}{2} \int_{0}^{1} A^{-}(\xi) \mathrm{d} \xi\right) D_{1}^{*} A^{+}(1) \exp \left(\theta \int_{0}^{1} A^{+}(\xi) \mathrm{d} \xi\right) D_{1} .\right.\right. \\
& \left.\left.\exp \left(-\frac{\theta}{2} \int_{0}^{1} A^{-}(\xi) \mathrm{d} \xi\right)-D_{1}^{*} A^{+}(1) D_{1}\right]\right\} \exp \left(\frac{\theta}{2} \int_{0}^{1} A^{-}(\xi) \mathrm{d} \xi\right) R^{-}(1, t) .
\end{aligned}
$$

In (2.9), the matrix in the square brackets is continuously differentiable and equal to zero for $\theta=0$. Thus, equation (2.3) implies that there is a $\theta_{1}>0$ such that for any $0<\theta<\theta_{1}$,

$$
R^{*}(1, t) \exp \left(\theta \int_{0}^{1} A(\xi) \mathrm{d} \xi\right) A(1) R(1, t) \leq-\frac{r^{-}}{2}\left\|\exp \left(\frac{\theta}{2} \int_{0}^{1} A^{-}(\xi) \mathrm{d} \xi\right) R^{-}(1, t)\right\|_{\mathbb{R}^{p}}^{2} .
$$

For the last term in (2.7), we can write

$$
\begin{aligned}
& R^{*}(x, t)\left(2 B(x)-A_{x}(x)\right) \exp \left(\theta \int_{0}^{x} A(\xi) \mathrm{d} \xi\right) R(x, t) \\
& =R^{*}(x, t) \exp \left(\frac{\theta}{2} \int_{0}^{x} A(\xi) \mathrm{d} \xi\right)\left(2 B(x)-A_{x}(x)\right) \exp \left(\frac{\theta}{2} \int_{0}^{x} A(\xi) \mathrm{d} \xi\right) R(x, t) \\
& +R^{*}(x, t) \exp \left(\frac{\theta}{2} \int_{0}^{x} A(\xi) \mathrm{d} \xi\right) G(\theta, x) \exp \left(\frac{\theta}{2} \int_{0}^{x} A(\xi) \mathrm{d} \xi\right) R(x, t),
\end{aligned}
$$

where

$$
G(\theta, x)=\exp \left(-\frac{\theta}{2} \int_{0}^{x} A(\xi) \mathrm{d} \xi\right)\left(2 B(x)-A_{x}(x)\right) \exp \left(\frac{\theta}{2} \int_{0}^{x} A(\xi) \mathrm{d} \xi\right)-\left(2 B(x)-A_{x}(x)\right) .
$$

Note that for any $w \in \mathbb{R}^{n}, w^{*} G(\theta, x) w$ is continuously differentiable with respect to $\theta$, and

$$
\begin{aligned}
w^{*} G(0, x) w & =0, \\
w^{*} \frac{\partial}{\partial \theta} G(0, x) w & =0 .
\end{aligned}
$$


(We have used the fact that $B(x)$ is symmetric in order to prove (2.14).) Using the last conditions and integrating by parts we can write

$$
w^{*} G(\theta, x) w=\int_{0}^{\theta}(\theta-\eta) \frac{\partial^{2}}{\partial \eta^{2}}\left(w^{*} G(\eta, x) w\right) \mathrm{d} \eta, \text { for all } w \in \mathbb{R}^{n}
$$

Then, for any $w \in \mathbb{R}^{n}$,

$$
w^{*} G(\theta, x) w \leq \frac{\theta^{2}}{2}\|w\|_{\mathbb{R}^{n}}^{2} \sup _{\eta \in[0, \theta]}\left\|\frac{\partial^{2}}{\partial \eta^{2}} G(\eta, x)\right\|_{\mathcal{L}\left(\mathbb{R}^{n}\right)} .
$$

From $(2.12)$, there is a $K_{1}>0$ such that

$$
\sup _{\eta \in[0, \theta]}\left\|\frac{\partial^{2}}{\partial \eta^{2}} G(\eta, x)\right\|_{\mathcal{L}\left(\mathbb{R}^{n}\right)} \leq K_{1}
$$

for any $x \in[0,1]$ and $0<\theta \leq \theta_{1}$. Substituting $(2.2,2.12,2.16)$ and (2.17) into (2.11) yields

$$
R^{*}(x, t)\left(2 B(x)-A_{x}(x)\right) \exp \left(\theta \int_{0}^{x} A(\xi) \mathrm{d} \xi\right) R(x, t) \leq K_{1} \theta^{2}\left\|\exp \left(\frac{\theta}{2} \int_{0}^{x} A(\xi) \mathrm{d} \xi\right) R(x, t)\right\|_{\mathbb{R}^{n}}^{2} .
$$

Substituting $(2.8,2.10)$ and $(2.18)$ into $(2.7)$ gives

$$
\frac{\mathrm{d} V_{\theta}(R(\cdot, t))}{\mathrm{d} t} \leq-\theta\left(\lambda_{\min }^{2}-K_{1} \theta\right) V_{\theta}(R(\cdot, t)),
$$

where $\lambda_{\min }^{2}=\min _{x \in[0,1], 1 \leq k \leq n} \lambda_{i}^{2}(x)$, which is positive by H.2. Choose $0<\theta^{*}<\theta_{1}$ such that $\lambda_{\text {min }}^{2}-K_{1} \theta^{*}$ $\geq \lambda_{\min }^{2} / 2$. It follows from $(2.19)$ that

$$
\frac{\mathrm{d} V_{\theta^{*}}(R(\cdot, t))}{\mathrm{d} t} \leq-\left(\frac{\theta^{*} \lambda_{\min }^{2}}{2}\right) V_{\theta^{*}}(R(\cdot, t)),
$$

or,

$$
V_{\theta^{*}}(R(\cdot, t)) \leq \exp \left(-t \theta^{*} \lambda_{\min }^{2} / 2\right) V_{\theta^{*}}\left(R_{0}\right)
$$

The last estimate implies exponential stability of the semigroup from the fact that $\sqrt{V_{\theta^{*}}(\cdot)}$ defines an equivalent norm on $X$.

Remark 3. Although we have not been able to prove the general Theorem 1 of Rauch and Taylor using our Lyapunov functional, the direct method of Lyapunov allows us to attack symmetric hyperbolic systems in higher dimension and degenerate hyperbolic cases that the theory in [25] cannot treat. In the sequel we present some examples to address the issue of possible applications. Because the mathematical complexity goes beyond the scope of the paper, the stability problem of symmetric hyperbolic systems in higher dimension will be presented elsewhere. However, the essential ideas are presented in the following examples:

Example 1. The hyperbolic system we consider describes the dynamic of temperatures $\tau_{i}(x, t)(i=1, \ldots, 4)$ in a classical countercurrent heat exchanger when the diffusion effect is neglected both in the fluids and in the separation wall ( $c f . \mathrm{Xu}$ and Gauthier [35]). The system is governed by

$$
\frac{\partial}{\partial t} \tau(x, t)=\Lambda \frac{\partial}{\partial x} \tau(x, t)+\Xi \tau(x, t), \quad(x, t) \in(0,1) \times \mathbb{R}^{+}
$$


where

$$
\tau=\left(\begin{array}{c}
\tau_{1} \\
\tau_{2} \\
\tau_{3} \\
\tau_{4}
\end{array}\right), \Lambda=\operatorname{diag}\left(-\rho_{1}, 0, \rho_{2}, 0\right), \Xi=\left(\begin{array}{cccc}
-h_{1} & h_{1} & 0 & 0 \\
h_{2} & -\left(h_{2}+h_{3}\right) & h_{3} & 0 \\
0 & h_{4} & -\left(h_{4}+h_{5}\right) & h_{5} \\
0 & 0 & h_{6} & -h_{6}
\end{array}\right)
$$

Note that all the constants $\rho_{1}, \rho_{2}$ and $h_{j}(j=1, \ldots, 4)$ are positive without any other constraint. The boundary and initial conditions are given by

$$
\tau_{1}(0, t)=\tau_{3}(1, t)=0, \quad \tau(x, 0)=\tau^{0}(x) .
$$

It is important to make the following remark. (i) $\Lambda$ is singular because it has two eigenvalues equal to zero. Hence we call the system degenerate. In [25] Rauch and Taylor have assumed $\Lambda$ to be nonsingular when the method of characteristics has been used. (ii) $\Xi$ is also singular. Because of the properties (i, ii) direct application of Theorem 1 is not possible. However, using an appropriate Lyapunov functional we can still establish the following result. Let $X=\left(L^{2}(0,1)\right)^{4}$.

Theorem 2. The system (2.21) is exponentially stable on the state space $X$.

Proof of Theorem 2. First, by some nonsingular diagonal matrix transformation we put the matrix $\Xi$ into the following symmetric form while $\Lambda$ is invariant (see [35]):

$$
\Xi=\left(\begin{array}{cccc}
-h_{1} & \sqrt{h_{1} h_{2}} & 0 & 0 \\
\sqrt{h_{1} h_{2}} & -\left(h_{2}+h_{3}\right) & \sqrt{h_{3} h_{4}} & 0 \\
0 & \sqrt{h_{3} h_{4}} & -\left(h_{4}+h_{5}\right) & \sqrt{h_{5} h_{6}} \\
0 & 0 & \sqrt{h_{5} h_{6}} & -h_{6}
\end{array}\right)
$$

It is easy to see that the linear operator $A=\Lambda \frac{\partial}{\partial x}+\Xi$ with domain $\mathcal{D}(A)=\left\{f \in\left(H^{1}(0,1)\right)^{4} \mid f_{1}(0)=f_{3}(1)=0\right\}$ is the generator of a $C_{0}$ semigroup of contractions on $X$. Let us consider the Lyapunov functional $V_{\theta}: X \rightarrow \mathbb{R}^{+}$ defined as follows:

$$
V_{\theta}(\phi)=\int_{0}^{1} \phi^{*}(x) \mathrm{e}^{x \theta \Lambda} \phi(x) \mathrm{d} x .
$$

Take a smooth initial condition $\tau^{0}$ in $X$. By computing the time derivative of the Lyapunov functional along the trajectory we obtain

$$
\begin{aligned}
\dot{V}_{\theta}(\tau)= & \left.\tau(x, t)^{*} \Lambda \mathrm{e}^{x \theta \Lambda} \tau(x, t)\right|_{0} ^{1}-\int_{0}^{1} \tau(x, t)^{*} \mathrm{e}^{(x / 2) \theta \Lambda}\left[\theta \Lambda^{2}-2 \Xi\right] \mathrm{e}^{(x / 2) \theta \Lambda} \tau(x, t) \mathrm{d} x \\
& +\int_{0}^{1} \tau(x, t)^{*} \mathrm{e}^{(x / 2) \theta \Lambda} \Pi(x, \theta) \mathrm{e}^{(x / 2) \theta \Lambda} \tau(x, t) \mathrm{d} x,
\end{aligned}
$$

where

$$
\Pi(x, \theta)=\mathrm{e}^{(x / 2) \theta \Lambda} \Xi \mathrm{e}^{-(x / 2) \theta \Lambda}+\mathrm{e}^{-(x / 2) \theta \Lambda} \Xi \mathrm{e}^{(x / 2) \theta \Lambda}-2 \Xi .
$$

In (2.24) the first term is negative by the boundary condition. By (2.23) there exists some large constant $m>1$ such that

$$
z^{*}\left(\theta \Lambda^{2}-2 \Xi\right) z \geq \frac{\theta}{m} z^{*} z, \quad \forall z \in \mathbb{R}^{4}, \forall \theta \in(0,1)
$$


From (2.25) we have some constant $\kappa>0$ such that

$$
\int_{0}^{1} \tau(x, t)^{*} \mathrm{e}^{(x / 2) \theta \Lambda} \Pi(x, \theta) \mathrm{e}^{(x / 2) \theta \Lambda} \tau(x, t) \mathrm{d} x \leq \kappa \theta^{2} V_{\theta}(\tau), \quad \forall \theta \in(0,1) .
$$

Substituting (2.26) and (2.27) into (2.24) we can find some small $\theta \in(0,1)$ such that

$$
\dot{V}_{\theta}(\tau) \leq-\frac{\theta}{2 m} V_{\theta}(\tau)
$$

The last inequality implies exponential stability of the system (2.21).

Example 2. In this example we consider a class of dissipative (symmetric) hyperbolic systems with several space variables. Using the Lyapunov method we propose a sufficient condition that guarantees exponential stability for this class of systems. We also give an numeric example of the class. Let $\Omega$ be a bounded open and connected subset in $\mathbb{R}^{m}$. We assume that the boundary $\partial \Omega$ is $C^{\infty}$. Let $S(x)$ denote a linear subspace of constant dimension in $\mathbb{R}^{N}$ which is smoothly varying on $\partial \Omega$. We consider the linear differential operator

$$
L=\sum_{j=1}^{m} L_{j}(x) \partial_{j}+\Xi(x)
$$

where $\partial_{j}$ denotes the partial derivative w.r.t. $x_{j}$ and all $L_{j}(x), \Xi(x) \in \mathbb{R}^{N \times N}$ are supposed to be $C^{\infty}(\bar{\Omega})$. We assume that all $L_{j}(x)$ are symmetric and the linear operator $L$ is formally dissipative, i.e., the following matrix is non-positive on $\Omega$ :

$$
\Xi^{*}(x)+\Xi(x)-\sum_{j=1}^{m} \partial_{j} L_{j}(x) \leq 0, \quad \forall x \in \Omega
$$

We set $n(x)=\left(n_{1}(x), \ldots, n_{m}(x)\right)$ equal to the outer unit normal to $\Omega$ at $x \in \partial \Omega$. The boundary matrix $\ell$ is defined by

$$
\ell(x)=\sum_{j=1}^{m} n_{j}(x) L_{j}(x), \quad \forall x \in \partial \Omega
$$

For the simplicity we assume $\ell$ to be non-singular at all points of $\partial \Omega$. We take $S$ to be maximal non-positive, i.e.,

$$
u^{*} \ell(x) u \leq 0, \quad \forall u \in S(x), \forall x \in \partial \Omega,
$$

and $S(x)$ is not properly contained in any other subspace having this property. Define the domain $\mathcal{D}(L)$ as follows:

$$
\mathcal{D}(L)=\left\{f=\left(f_{1}, \ldots, f_{N}\right)^{*} \mid f_{j} \in C^{1}(\Omega) \cap C(\bar{\Omega}) ; f(x) \in S(x), \forall x \in \partial \Omega\right\} .
$$

Let $X=\left(L^{2}(\Omega)\right)^{N}$. Using density of $C_{0}^{\infty}(\Omega)$ in $L^{2}(\Omega)$ and the Green formula in $\langle L f, g\rangle_{X}$ we can prove the following result:

Lemma 1. The linear operator $L$ is closable, i.e., it has a unique closed extension noted by $\widetilde{L}$.

Lemma 2. The operator $\widetilde{L}$ is the generator of a $C_{0}$ semigroup of contractions on $X$. 
Proof of Lemma 2. We prove first that $\widetilde{L}$ is dissipative and then that $(I-\widetilde{L})$ is surjective. Hence $\widetilde{L}$ is the generator of a $C_{0}$ semigroup of contractions on $X$. Since $L$ is formally dissipative and $S$ is non-positive, for all $f \in \mathcal{D}(L)$ the following holds:

$$
\langle\widetilde{L} f, f\rangle_{X} \leq 0
$$

Since $\mathcal{D}(L)$ is a core of $\mathcal{D}(\widetilde{L})$, the inequality $(2.29)$ remains true for all $f \in \mathcal{D}(\widetilde{L})$. Hence $\widetilde{L}$ is dissipative. Given $g \in X$ we look for some $f \in \mathcal{D}(\widetilde{L})$ such that

$$
(I-\widetilde{L}) f=g
$$

From Lax and Phillips [15] (see also Chen [3]) we know that for each $g \in X$, there exists some strongly converging sequence $\left(f_{n}\right)$ in $\mathcal{D}(L)$ such that $\left(L f_{n}\right)$ converges strongly to $g$. By definition the limit $f$ of $\left(f_{n}\right)$ belongs to $\mathcal{D}(\widetilde{L})$ and satisfies the equation $(2.30)$. Hence $(I-\widetilde{L})$ is surjective.

Now we consider the evolution system on $X$

$$
\left\{\begin{array}{l}
\dot{\varphi}(t)=\widetilde{L} \varphi(t) \\
\varphi(0)=\varphi^{0}
\end{array}\right.
$$

The main result in this Example 2 is the following:

Theorem 3. Suppose that all the above assumptions are satisfied. Then the semigroup generated by $\widetilde{L}$ is exponentially stable if there exist some constants $\theta>0$ and $\alpha_{j} \in \mathbb{R}, j=1, \ldots, m$, such that

$$
D(x, \theta, \alpha)=\Xi^{*}(x)+\Xi(x)-\sum_{j=1}^{m} \partial_{j} L_{j}(x)-\theta \sum_{j=1}^{m} \alpha_{j} L_{j}(x)<0, \quad \forall x \in \bar{\Omega} .
$$

Proof of Theorem 3. Taking $\varphi^{0} \in \mathcal{D}(\widetilde{L})$ we have $\varphi \in C^{1}([0, \infty) ; X)$. Consider the Lyapunov functional $V_{\theta}$ : $X \rightarrow \mathbb{R}^{+}$such that

$$
V_{\theta}(\varphi(t))=\int_{\Omega} \varphi^{*}(t) w(\theta, x) \varphi(t) \mathrm{d} x
$$

where $w(\theta, x)=\exp \left(\theta \sum_{k=1}^{m} \alpha_{k} x_{k}\right)$. Differentiating the functional along the trajectory of (2.31) gives us

$$
\dot{V}(\varphi(t))=\int_{\Omega}\left\{(\widetilde{L} \varphi)^{*} w(\theta, x) \varphi+\varphi^{*} w(\theta, x) \widetilde{L} \varphi\right\} \mathrm{d} x
$$

For each $t$ the computation of the last integral is carried out on some sequence in $\mathcal{D}(L)$ which approximates $\varphi(t)$ in the sense of the graph topology. Then we pass it to limit. Using the fact that $S$ is non-positive it is easy to see that

$$
\dot{V}(\varphi(t)) \leq \int_{\Omega} \varphi^{*} w(\theta, x) D(x, \theta, \alpha) \varphi \mathrm{d} x
$$

If the condition (2.32) is satisfied then (2.33) implies exponential stability of the semigroup.

A numeric example is given by the open disc in $\mathbb{R}^{2}$ :

$$
\Omega=\left\{x \in \mathbb{R}^{2} \mid x_{1}^{2}+x_{2}^{2}<1\right\} .
$$


Let $X=\left(L^{2}(\Omega)\right)^{2}$ (equipped with the usual inner product: $\left.\int_{\Omega} f^{*}(x) g(x) \mathrm{d} x\right)$. Consider the following matrices:

$$
G=\left(\begin{array}{cc}
x_{2}+1 & x_{1} \\
x_{1} & -x_{2}+1
\end{array}\right), \quad L_{1}=\left(\begin{array}{ll}
0 & 1 \\
1 & 0
\end{array}\right), \quad L_{2}=\left(\begin{array}{cc}
1 & 0 \\
0 & -1
\end{array}\right), \quad \Xi=\left(\begin{array}{cc}
0 & 0 \\
0 & -c
\end{array}\right)
$$

Let $S(x)$ be the subspace in $\mathbb{R}^{2}$ given by $S(x)=\operatorname{Ker}(G)$. The boundary matrix $\ell(x)=\left(\begin{array}{cc}x_{2} & x_{1} \\ x_{1} & -x_{2}\end{array}\right)$ has two eigenvalues: $\lambda_{1}=-1$ and $\lambda_{2}=1$ on $\partial \Omega$. Moreover the two corresponding eigenspaces are orthogonal to each other. The maximal subspace of $u$ in $\mathbb{R}^{2}$ satisfying $(2.28)$ is the eigenspace corresponding to $\lambda_{1}$. Hence $S(x)$ is maximal and non-positive. Since all the conditions required by Theorem 3 are satisfied, the numeric system is exponentially stable when $\min _{x \in \bar{\Omega}} c(x)>0$.

Remark 4. Assume our hypothesis satisfied. For each $\varphi^{0} \in L^{2}(\Omega)$ there exists a unique $\varphi \in L^{2}((0, T) \times \Omega)$ which satisfies the equation (2.33) in the weak sense in $L^{2}((0, T) \times \Omega), \forall T>0$ (cf. Rauch [24]). In addition $\varphi \in C\left([0, \infty) ; L^{2}(\Omega)\right)$ and converges exponentially to zero in $L^{2}(\Omega)$ as $t \rightarrow \infty$. However the regularity for the solutions of (2.33) has not been addressed here. The interested reader is referred to Lax and Phillips [15] and to Rauch [24] for more general situations.

\section{Exponential STABILITy AND WELL-POSEDNESS OF THE NETWORK}

In this section we show that the heat exchanger network (1.1-1.3) can be transformed into the form (2.1). We prove exponential stability of the system by applying Theorem 1 . Then we show that the system is well-posed.

\subsection{Exponential stability of the network}

Now, we show how to transform the heat exchanger system (1.1-1.4) into the form (2.1). First, the following transformation allows to normalize the space variable to the interval $[0,1]$ :

$$
\phi(x, t)=\left(\begin{array}{l}
\phi_{1}(x, t) \\
\phi_{2}(x, t) \\
\phi_{3}(x, t) \\
\phi_{4}(x, t)
\end{array}\right)=\left(\begin{array}{l}
R_{2}\left(l_{1} x, t\right) \\
T_{1}\left(\left(l_{1}-l_{2}\right) x+l_{2}, t\right) \\
R_{1}\left(l_{1} x, t\right) \\
T_{2}\left(\left(l_{1}-l_{2}\right) x+l_{2}, t\right)
\end{array}\right)
$$

Then the system $(1.1-1.4)$ is equivalent to

$$
\left\{\begin{array}{l}
\frac{\partial \phi(x, t)}{\partial t}=A_{1} \frac{\partial \phi(x, t)}{\partial x}+B_{1} \phi(x, t)+b(x) u(t), \quad(x, t) \in(0,1) \times \mathbb{R}^{+} \\
\left(\begin{array}{c}
\phi_{1} \\
\phi_{2}
\end{array}\right)(0, t)=\left(\begin{array}{l}
1 \\
0
\end{array}\right) d(t) \\
\left(\begin{array}{c}
\phi_{3} \\
\phi_{4}
\end{array}\right)(1, t)=\left(\begin{array}{cc}
0 & 1-\beta \\
1 & 0
\end{array}\right)\left(\begin{array}{l}
\phi_{1} \\
\phi_{2}
\end{array}\right)(1, t) \\
y(t)=\phi_{4}(0, t),
\end{array}\right.
$$

where $A_{1}$ and $B_{1}$ are constant matrices:

$$
A_{1}=\operatorname{diag}\left(-\frac{m_{2}}{l_{1}},-\frac{(1-\beta) m_{1}}{l_{2}-l_{1}}, \frac{m_{1}}{l_{1}}, \frac{m_{2}}{l_{2}-l_{1}}\right)
$$




$$
B_{1}=\left(\begin{array}{cccc}
-K_{2} & 0 & K_{2} & 0 \\
0 & -K_{1} & 0 & K_{1} \\
K_{1} & 0 & -K_{1} & 0 \\
0 & K_{2} & 0 & -K_{2}
\end{array}\right),
$$

and

$$
b(x)=\left(0, b_{2}\left(\left(l_{1}-l_{2}\right) x+l_{2}\right), b_{1}\left(l_{1} x\right), 0\right)^{*} .
$$

Note that $B_{1}$ is not symmetric in (3.4). Set

$$
\varpi=\sqrt{\frac{l_{1}}{\left(l_{2}-l_{1}\right)(1-r)}} .
$$

The following linear (diagonal) transformation $\Lambda$ applied to (3.2) makes $B_{1}$ symmetric by keeping $A_{1}$ unchanged:

$$
\Lambda\left(\phi_{1}, \phi_{2}, \phi_{3}, \phi_{4}\right)=\left(\varpi \phi_{1}, \sqrt{\frac{K_{2}}{K_{1}}} \phi_{2}, \varpi \sqrt{\frac{K_{2}}{K_{1}}} \phi_{3}, \phi_{4}\right),
$$

where $r \in[0, \beta]$ is an arbitrary constant such that the boundary condition becomes dissipative (see (2.3) and (3.13) below). We set

$$
\begin{gathered}
B_{2}=\Lambda B_{1} \Lambda^{-1}=\left(\begin{array}{cccc}
-K_{2} & 0 & \sqrt{K_{1} K_{2}} & 0 \\
0 & -K_{1} & 0 & \sqrt{K_{1} K_{2}} \\
\sqrt{K_{1} K_{2}} & 0 & -K_{1} & 0 \\
0 & \sqrt{K_{1} K_{2}} & 0 & -K_{2}
\end{array}\right), \\
\widetilde{b}_{1}(x)=\left(0, \sqrt{K_{2} / K_{1}} b_{2}\left(\left(l_{1}-l_{2}\right) x+l_{2}\right), \varpi \sqrt{K_{2} / K_{1}} b_{1}\left(l_{1} x\right), 0\right)^{*}, \\
\widetilde{b}_{2}=\varpi\left(\begin{array}{c}
1 \\
0
\end{array}\right),
\end{gathered}
$$

and

$$
\tilde{D}_{1}=\left(\begin{array}{cc}
0 & (1-\beta) \varpi \\
\varpi^{-1} & 0
\end{array}\right) .
$$

Keeping the same notation, the transformation (3.6) applied to (3.2) leads to:

$$
\left\{\begin{array}{l}
\frac{\partial \phi(x, t)}{\partial t}=A_{1} \frac{\partial \phi(x, t)}{\partial x}+B_{2} \phi(x, t)+\widetilde{b}_{1}(x) u(t), \quad(x, t) \in(0,1) \times \mathbb{R}^{+} \\
\left(\begin{array}{l}
\phi_{1} \\
\phi_{2}
\end{array}\right)(0, t)=\widetilde{b}_{2} \cdot d(t) \\
\left(\begin{array}{c}
\phi_{3} \\
\phi_{4}
\end{array}\right)(1, t)=\tilde{D}_{1}\left(\begin{array}{c}
\phi_{1} \\
\phi_{2}
\end{array}\right)(1, t) \\
y(t)=\phi_{4}(0, t) .
\end{array}\right.
$$

We obtain a control system with two inputs and an output. The state space is $X=\left(L^{2}(0,1)\right)^{4}$ with the corresponding inner product. We denote by $U$ and $Y$ the control space and the observation space: $U=\mathbb{R}^{2}$ and 
$Y=\mathbb{R}$, respectively. Let $\mathcal{A}$ be the unbounded operator defined by

$$
\mathcal{D}(\mathcal{A})=\left\{f \in\left(H^{1}(0,1)\right)^{4} \mid\left(\begin{array}{l}
f_{1} \\
f_{2}
\end{array}\right)(0)=0,\left(\begin{array}{l}
f_{3} \\
f_{4}
\end{array}\right)(1)=\tilde{D}_{1}\left(\begin{array}{l}
f_{1} \\
f_{2}
\end{array}\right)(1)\right\}
$$

and for each $f \in \mathcal{D}(\mathcal{A})$,

$$
\mathcal{A} f(x)=A_{1} \frac{\partial f(x)}{\partial x}+B_{2} f(x)
$$

Theorem 4. The operator $\mathcal{A}$ is the generator of a $C_{0}$-semigroup (denoted $\mathrm{e}^{\text {t } \mathcal{A}}$ ) of contractions on $X$ which is exponentially stable.

Proof of Theorem 4. The conditions H.1, H.2, (2.2) and (2.4) in H.3 are satisfied with $r^{+}>0$ because $B_{2}$ is dissipative and $D_{0}=0$. The condition (2.3) is also satisfied for all $0 \leq r \leq \beta$ :

$$
A_{1}^{-}+\tilde{D}_{1}^{*} A_{1}^{+} \tilde{D}_{1}=\left(\begin{array}{cc}
-\frac{r m_{2}}{l_{1}} & 0 \\
0 & -\frac{(1-\beta) m_{1}(\beta-r)}{\left(l_{2}-l_{1}\right)(1-r)}
\end{array}\right) \leq 0
$$

The statement now follows from Theorem 1.

Remark 5. From Theorem 4, zero is in the resolvent set $\rho(\mathcal{A})$ and the growth bound $\omega_{0}(\mathcal{A})$ of the considered semigroup is negative. Recall that

$$
\omega_{0}(\mathcal{A})=\lim _{t \rightarrow+\infty} t^{-1} \ln \left\|\mathrm{e}^{t \mathcal{A}}\right\|
$$

Now we recall some standard notation that we will use in the following sections. Suppose that $0 \in \rho(\mathcal{A})$, which is the case for (2.1). We define the Hilbert spaces $X_{1}$ and $X_{-1}$ as follows: $X_{1}$ is $\mathcal{D}(\mathcal{A})$ with the norm $\|f\|_{1}=\|\mathcal{A} f\|_{X}$ and $X_{-1}$ is the completion of $X$ with respect to the norm $\|f\|_{-1}=\left\|\mathcal{A}^{-1} f\right\|_{X}$. We have $X_{1} \subset X \subset X X_{-1}$, densely and with continuous embeddings. The operator $\mathcal{A}$ has a unique extension on the whole space $X$ because it is defined on a dense set $\mathcal{D}(\mathcal{A})$ in $X$ and continuous from $X$ to $X_{-1}$. The semigroup e $\mathrm{e}^{t \mathcal{A}}$ can be extended to a $C_{0}$-semigroup on $X_{-1}$ whose generator is the extended operator $\mathcal{A} \in \mathcal{L}\left(X, X_{-1}\right)$. Let $\mathcal{D}\left(\mathcal{A}^{*}\right)$ be the Hilbert space normed with $\left\|\mathcal{A}^{*} \cdot\right\|_{X}$. We define the duality product on $X_{-1} \times \mathcal{D}\left(\mathcal{A}^{*}\right)$ by continuous extension of the inner product on $X$ : For all $h \in X$ and all $g \in \mathcal{D}\left(\mathcal{A}^{*}\right),\langle h, g\rangle_{X_{-1}, \mathcal{D}\left(\mathcal{A}^{*}\right)}=\langle h, g\rangle_{X}$. The mapping $J: X_{-1} \rightarrow \mathcal{D}^{\prime}\left(\mathcal{A}^{*}\right)$ (topological dual of $\mathcal{D}\left(\mathcal{A}^{*}\right)$ ) such that $J h(f)=\langle h, f\rangle_{X_{-1}, \mathcal{D}\left(\mathcal{A}^{*}\right)}$ is an isomorphism. Moreover, it is easy to see that for all $f \in X$ and all $g \in \mathcal{D}\left(\mathcal{A}^{*}\right)$,

$$
\left\langle\mathrm{e}^{t \mathcal{A}} \mathcal{A} f, g\right\rangle_{X_{-1}, \mathcal{D}\left(\mathcal{A}^{*}\right)}=\left\langle f, \mathrm{e}^{t \mathcal{A}^{*}} \mathcal{A}^{*} g\right\rangle_{X}, \quad \forall t \geq 0 .
$$

For each $\alpha \in \mathbb{R}, \mathbb{C}_{\alpha}$ denotes the open right half-plane defined by

$$
\mathbb{C}_{\alpha}=\{\lambda \in \mathbb{C} \mid \Re e(\lambda)>\alpha\}
$$

\subsection{Well-posedness of the network with control and observation}

In this subsection we prove that the system (3.11) is well-posed in the sense of Salamon [28] and Weiss [31]. Roughly speaking the system is well-posed if the following mappings are continuous for all $T>0$ : the control-to-state mapping $(u, d) \in L^{2}((0, T) ; U) \rightarrow \phi(\cdot, T) \in X$, the state-to-observation mapping $\phi_{0} \in X \rightarrow y$ $\in L^{2}((0, T) ; Y)$ and the control-to-observation mapping $(u, d) \in L^{2}((0, T) ; U) \rightarrow y \in L^{2}((0, T) ; Y)$. For an 
abstract definition of well-posed systems see, for example [28,31] and [33]. Although the proof is presented for our particular system, our method is valid for symmetric hyperbolic systems of the form (2.1).

Since the system is well-posed, there is a unique transfer function $H(s)$

$$
H(s)=[P(s), W(s)]
$$

which is analytic and bounded on some open right half-plane $\mathbb{C}_{\alpha}$. As our semigroup is exponentially stable, according to the general theory developed in [32] we know that $\alpha$ must be negative. Hence our transfer functions $P(s)$ and $W(s)$ are analytic and bounded on the closed right half-plane. This result is useful for controller design in the frequency domain (see $[7,8]$ and $[10]$ ).

Note that the system (3.11) has an input which is via boundary control and that the observation is on the boundary. In order to represent such a system we need to consider the adjoint operator $\mathcal{A}^{*}$ of $\mathcal{A}$. Let $\mathcal{A}^{*}$ be defined by

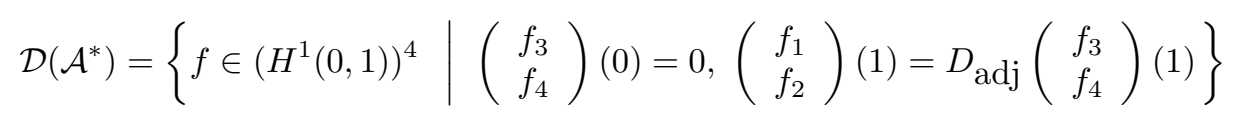

where

$$
D_{\mathrm{adj}}=-\left(A_{1}^{-}\right)^{-1} \widetilde{D}_{1}^{*} A_{1}^{+}=\left(\begin{array}{cc}
0 & \sqrt{\frac{l_{1}(1-r)}{l_{2}-l_{1}}} \\
\sqrt{\frac{l_{2}-l_{1}}{l_{1}(1-r)}} & 0
\end{array}\right)
$$

and for each $f \in \mathcal{D}\left(\mathcal{A}^{*}\right)$,

$$
\mathcal{A}^{*} f(x)=-A_{1} \frac{\partial f(x)}{\partial x}+B_{2} f(x)
$$

Define the observation operator $C_{\text {adj }} \in \mathcal{L}\left(\mathcal{D}\left(\mathcal{A}^{*}\right), Y\right)$ such that $C_{\text {adj }} f=\varrho \cdot f_{1}(0)$, where $\varrho=m_{2}\left[(1-r) l_{1}\left(l_{2}\right.\right.$ $\left.\left.-l_{1}\right)\right]^{-1 / 2}$. Therefore the linear form $C_{\mathrm{adj}}\left(\mathcal{A}^{*}\right)^{-1}$ is continuous from $X$ to $\mathbb{R}$. From the representation theorem of Riesz there is a unique element $\xi_{\mathrm{adj}} \in X$ such that $C_{\mathrm{adj}}\left(\mathcal{A}^{*}\right)^{-1} f=\left\langle\xi_{\mathrm{adj}}, f\right\rangle_{X}$ for all $f \in X$. We claim that $\xi_{\text {adj }} \in\left(H^{1}(0,1)\right)^{4}$. The interested reader is referred to the Appendix for a proof. Of course the adjoint semigroup $\mathrm{e}^{t \mathcal{A}^{*}}$ is exponentially stable.

We consider the dual system corresponding to (3.11) (with $u=0$ and without observation) as follows:

$$
\left\{\begin{array}{l}
\frac{\partial \psi}{\partial t}=-\mathcal{A}^{*} \psi \\
\psi(\cdot, T)=\psi_{0} \\
y_{\mathrm{adj}}(t)=\left(C_{\mathrm{adj}} \psi\right)(t)
\end{array}\right.
$$

Let $U_{2}$ be the control space for the second component of the control: $U_{2}=\mathbb{R}$. We define the state-to-observation mapping $\Theta_{T}: X \rightarrow L^{2}\left((0, T) ; U_{2}\right)$ such that for all $\psi_{0} \in \mathcal{D}\left(A^{*}\right)$,

$$
\Theta_{T} \psi_{0}(t)=C_{\mathrm{adj}} \mathrm{e}^{(T-t) \mathcal{A}^{*}} \psi_{0}
$$

Lemma 3. The linear mapping $\Theta_{T}$ is continuous for each $T>0$ : there exist some positive constants $K>0$ and $\gamma>0$ such that

$$
\left\|\Theta_{T}\right\|_{\mathcal{L}\left(X, L^{2}\left((0, T) ; U_{2}\right)\right)} \leq K \mathrm{e}^{T \gamma}
$$


Proof of Lemma 3. Since the domain of $\left(\mathcal{A}^{*}\right)^{n}$ is dense in $X$ for any $n \in \mathbb{N}$, we take $\psi_{0}$ such that $\psi(\cdot, t)$ $=\mathrm{e}^{(T-t) \mathcal{A}^{*}} \psi_{0}$ is the strong solution of (3.18). Denote by $\lambda_{k}, k=1,2, \ldots, 4$ the eigenvalues of $A_{1}$ in (3.3). Hence $\lambda_{k}<0$ and $\lambda_{k+2}>0, k=1,2$. We take the inner product of the both sides in (3.18) with the multiplier

$$
m(x, t)=\left[(1-x) \cdot \psi_{1}(x, t),(1-x) \cdot \psi_{2}(x, t), x \cdot \psi_{3}(x, t), x \cdot \psi_{4}(x, t)\right]^{*} .
$$

Integrating by parts on $(0,1) \times(0, T)$ we get

$$
\int_{0}^{T} \mathcal{Y}(t) \mathrm{d} t=\int_{0}^{1}[\mathcal{Z}(x, T)-\mathcal{Z}(x, 0)] \mathrm{d} x+\int_{0}^{T} \int_{0}^{1} \mathcal{Q}(x, t) \mathrm{d} x \mathrm{~d} t
$$

where

$$
\begin{gathered}
\mathcal{Y}(t)=\sum_{k=1}^{2}\left\{\lambda_{k+2} \psi_{k+2}^{2}(1, t)-\lambda_{k} \psi_{k}^{2}(0, t)\right\}, \\
\mathcal{Z}(x, t)=\sum_{k=1}^{2}\left\{(1-x) \cdot \psi_{k}^{2}(x, t)+x \cdot \psi_{k+2}^{2}(x, t)\right\}
\end{gathered}
$$

and

$$
\mathcal{Q}(x, t)=\sum_{k=1}^{2}\left\{\lambda_{k+2} \psi_{k+2}^{2}(x, t)-\lambda_{k} \psi_{k}^{2}(x, t)\right\}+2 m(x, t)^{*} B_{2} \psi(x, t)
$$

Since $\mathcal{A}^{*}$ is the generator of a $C_{0}$-semigroup the following holds for some constants $M \geq 1$ and $\gamma>0$ :

$$
\|\psi(\cdot, t)\| \leq M \mathrm{e}^{(T-t) \gamma}\left\|\psi_{0}\right\|
$$

Using (3.21) and the Cauchy inequality in (3.20) we obtain inequality (3.19), and so prove the lemma.

Define the observation operator $\mathcal{C} \in \mathcal{L}\left(X_{1}, Y\right)$ and the control operator $\mathcal{B}=\left[\mathcal{B}_{1} \mathcal{B}_{2}\right] \in \mathcal{L}\left(U, X_{-1}\right)$ such that $\mathcal{C} f=f_{4}(0)$ and $\left[\mathcal{B}_{1} \mathcal{B}_{2}\right]=\left[\begin{array}{ll}\widetilde{b}_{1} & A \xi_{\text {adj }}\end{array}\right]$, respectively. One of our main results is the following:

Theorem 5. System (3.11) is well-posed. Moreover, if the initial data $\phi_{0}=0$ then

$$
y(t)=\mathcal{C} \int_{0}^{t} \mathrm{e}^{(t-\tau) \mathcal{A}} \mathcal{B}\left[\begin{array}{l}
u(\tau) \\
d(\tau)
\end{array}\right] \mathrm{d} \tau, \quad \forall(u, d) \in C_{0}^{\infty}((0,+\infty) ; U),
$$

and if $(u, d)=0$ then $y(t)=\mathcal{C} \mathrm{e}^{t \mathcal{A}} \phi_{0}, \forall \phi_{0} \in \mathcal{D}(\mathcal{A})$.

Proof of Theorem 5. For $(u, d)=0$ we prove the continuity of the state-to-observation mapping as the same as in the proof of Lemma 3 (using the multiplier in (3.25)). To prove the continuity of the control-to-state mapping we take $\psi_{0}=0$ and $(u, d) \in C_{0}^{\infty}((0,+\infty) ; U)$ which is dense in $L^{2}((0, T) ; U)$. Since the first part of the control operator is bounded and the corresponding control-to-state mapping is continuous and bounded like (3.19). Hence we set $u=0$. We claim that for any $d \in C_{0}^{\infty}\left((0,+\infty) ; U_{2}\right)$, equation $(3.11)$ has a unique (classical) solution (see the definition in Pazy [21], pp. 105-110). (Recall that $U_{2}$ has been defined before Lem. 3.) Indeed, consider the following affine transformation

$$
\widetilde{\phi}(x, t)=\phi(x, t)-\left(\begin{array}{c}
(1-x) \cdot \tilde{b}_{2} \cdot d(t) \\
0
\end{array}\right) .
$$


Then $\widetilde{\phi}(x, t)$ satisfies the following PDE

$$
\left\{\begin{array}{l}
\frac{\partial \widetilde{\phi}}{\partial t}=\mathcal{A} \widetilde{\phi}+f(t) \\
\widetilde{\phi}(\cdot, 0)=0
\end{array}\right.
$$

where $f \in C_{0}^{\infty}((0,+\infty) ; X)$. It is easy to check that (3.22) has a unique solution and it is also a solution of (3.11). The uniqueness of solution is obvious for (3.11).

Taking the inner product $\int_{0}^{T}\langle\phi(\cdot, t), \psi(\cdot, t)\rangle_{X} \mathrm{~d} t$ in (3.18) and integrating by parts we obtain

$$
\left\langle\phi(\cdot, T), \psi_{0}\right\rangle_{X}=\left\langle d, \Theta_{T} \psi_{0}\right\rangle_{L^{2}\left((0, T) ; U_{2}\right)}, \forall \psi_{0} \in \mathcal{D}\left(\mathcal{A}^{*}\right) .
$$

Since $\mathcal{D}\left(\mathcal{A}^{*}\right)$ is dense in $X$, by Lemma 3 we have $\phi(\cdot, T)=\Theta_{T}^{*} d$. Hence the control-to-state mapping is bounded. Recalling that $\left\|\Theta_{T}^{*}\right\|=\left\|\Theta_{T}\right\|$ we get easily some constants $K \geq 1$ and $\gamma>0$ such that

$$
\|\phi(\cdot, T)\| \leq K \mathrm{e}^{\gamma T}\left\|\left(\begin{array}{l}
u \\
d
\end{array}\right)\right\|_{L^{2}((0, T) ; U)}, \quad \forall T \geq 0 .
$$

Using the standard notation introduced at the end of the last subsection with (3.23) we find the expression of $\phi(\cdot, T)$ for $d \in C_{0}^{\infty}\left((0,+\infty) ; U_{2}\right)$ :

$$
\phi(\cdot, T)=\int_{0}^{T} \mathrm{e}^{(t-\tau) \mathcal{A}} \mathcal{A} \xi_{\text {adj }} d(\tau) \mathrm{d} \tau
$$

The rest is to prove the continuity of the control-to-observation mapping. We apply to (3.11) the similar reasoning with the multiplier $m(x, t)$ as in the proof of Lemma 3

$$
m(x, t)=\left(\begin{array}{c}
x \cdot \phi^{-}(x, t) \\
(1-x) \cdot \phi^{+}(x, t)
\end{array}\right)
$$

Using (3.24) and the Cauchy inequality we prove the existence of some constants $K \geq 1$ and $\gamma>0$ such that

$$
\|y\|_{L^{2}((0, T) ; Y)} \leq K \mathrm{e}^{\gamma T}\left\|\left(\begin{array}{c}
u \\
d
\end{array}\right)\right\|_{L^{2}((0, T) ; U)} .
$$

Hence the system is well-posed.

\section{REgularity, transfer FUnCtions AND INNER-OUter FACTORIZATION}

A regular system is a well-posed system such that for every $\mathrm{v} \in U$, the following limit exists in $Y$ :

$$
\lim _{\substack{s \rightarrow+\infty \\ s \in \mathbb{R}}} H(s) \mathrm{v}=D \mathrm{v} .
$$

If the system is regular, $D \in \mathcal{L}(U, Y)$. One advantage of regular systems is that the transfer functions can be expressed in terms of the operators in the state equation as for finite-dimensional systems. As a consequence the well developed system and control theory in finite dimensions has been generalized for regulars systems (see $[17,18,29]$ and $[33])$. 
Let $\mathcal{C}_{\Lambda}$ be the $\Lambda$-extension of $\mathcal{C} \in \mathcal{L}\left(X_{1}, Y\right)$ defined by

$$
\mathcal{C}_{\Lambda} z=\lim _{\substack{\lambda \rightarrow+\infty \\ \lambda \in \mathbb{R}}} \lambda \cdot \mathcal{C}(\lambda I-\mathcal{A})^{-1} z .
$$

Hence $X_{1} \subset \mathcal{D}\left(\mathcal{C}_{\Lambda}\right) \subset X$, see Weiss [31] for more details.

Theorem 6. The system described by (3.11) is regular. Moreover its feedthrough operator D is zero and its transfer function $H(s)$ is given by

$$
H(s)=\mathcal{C}_{\Lambda}(s I-\mathcal{A})^{-1} \mathcal{B}
$$

for all $s \in \mathbb{C}$ with $\Re e(s)>\omega_{0}(\mathcal{A})$.

Proof of Theorem 6. The control-to-observation operator being a shift invariant bounded operator from $L^{2}([0,+\infty) ; U)$ to $L^{2}([0,+\infty) ; Y)$, there is a unique $\mathcal{L}(U, Y)$-valued function $H(s)$ analytic and bounded on $\mathbb{C}_{0}$ such that $\hat{y}(s)=H(s)[\hat{u}(s), \hat{d}(s)]^{*}$ for all $s \in \mathbb{C}_{0}$. If we prove $D=0$ in (4.1) then the system is regular. The identity (4.2) for a regular system follows from [31] and [32].

We sketch only the proof that $D=0$. Consider the simplified (diagonal) system with additional control and additional observation as follows:

$$
\left\{\begin{array}{l}
\frac{\partial \phi}{\partial t}=A_{1} \frac{\partial \phi}{\partial x}+\widetilde{b}_{1} u(t)+v(\cdot, t) \\
\left(\begin{array}{c}
\phi_{1} \\
\phi_{2}
\end{array}\right)(0, t)=\widetilde{b}_{2} \cdot d(t) \\
\left(\begin{array}{c}
\phi_{3} \\
\phi_{4}
\end{array}\right)(1, t)=\tilde{D}_{1}\left(\begin{array}{l}
\phi_{1} \\
\phi_{2}
\end{array}\right)(1, t) \\
\widetilde{y}(t)=\left(\begin{array}{l}
\phi_{4}(0, t) \\
\phi(\cdot, t)
\end{array}\right)
\end{array}\right.
$$

It is easy to see that the first part of Theorem 5 is valid for (4.3). System (3.11) can be seen as the closed-loop system of (4.3) with the output feedback $[u, d, v]^{*}=K \tilde{y}+[\tilde{u}, \tilde{d}, \tilde{v}]^{*}$, where

$$
K=\left(\begin{array}{ll}
0 & 0 \\
0 & B_{2}
\end{array}\right)
$$

We prove that (4.3) is regular with the feedthrough operator $\widetilde{D}=0$. We prove also that $K$ is an admissible feedback operator (see [31] for the definition) and $I-\tilde{D} \cdot K$ is invertible. Then (3.11) is regular and $D=0$ as easily computed by $D^{K}=(I-\tilde{D} \cdot K)^{-1} \tilde{D}$.

To prove $\widetilde{D}=0$ take the Laplace transform in (4.3). Then the obtained differential equation is explicitly solvable. Denote by $\widetilde{H}(s)$ the transfer function $(\hat{u}, \hat{d}, \hat{v}) \rightarrow \widehat{\widetilde{y}}$. It is easy to see that there is some constant $K>0$ such that the following holds:

$$
\sup _{\omega \in \mathbb{R}}\left\|\widetilde{H}(\sigma+i \omega) \cdot\left[\begin{array}{c}
\mathrm{u} \\
\mathrm{d} \\
\mathrm{v}
\end{array}\right]\right\|_{Y \times X} \leq\left(\frac{K}{\sigma}\right)\left\|\left[\begin{array}{c}
\mathrm{u} \\
\mathrm{d} \\
\mathrm{v}
\end{array}\right]\right\|_{U \times X}, \forall \sigma>0 .
$$

Using Proposition 4.9 of [31] one proves the theorem. We leave the details to the reader. 
The following result is a direct consequence of the regularity of system (3.11):

Proposition 1. System (3.11) can be written as follows:

$$
\left\{\begin{array}{l}
\frac{\mathrm{d} \phi(t)}{\mathrm{d} t}=\mathcal{A} \phi(t)+\mathcal{B}_{1} u(t)+\mathcal{B}_{2} d(t) \\
y(t)=\mathcal{C}_{\Lambda} \phi(t)
\end{array}\right.
$$

where the first equation holds in $X_{-1}$ for every $(u, d) \in L^{2}([0,+\infty) ; U)$ and for almost every $t \geq 0$, and the second holds in $Y$ for almost every $t \geq 0$.

Let $H^{\infty}\left(\mathbb{C}_{0}\right)$ be the Banach space of analytic functions on $\mathbb{C}_{0}$ such that for any $f \in H^{\infty}\left(\mathbb{C}_{0}\right)$,

$$
\|f\|_{\infty}=\sup _{\sigma>0} \sup _{\omega \in \mathbb{R}}|f(\sigma+i \omega)|<\infty
$$

Let $H^{2}\left(\mathbb{C}_{0}\right)$ be the Hilbert space of analytic functions on $\mathbb{C}_{0}$ such that for any $f \in H^{2}\left(\mathbb{C}_{0}\right)$,

$$
\|f\|_{2}^{2}=\sup _{\sigma>0} \int_{\mathbb{R}}|f(\sigma+i \omega)|^{2} \mathrm{~d} \omega<\infty
$$

An element $m$ in $H^{\infty}\left(\mathbb{C}_{0}\right)$ is called inner if $|m(s)| \leq 1$ on $\mathbb{C}_{0}$ and $|m(s)|=1$ a.e. on $i \mathbb{R}$. An element in $H^{\infty}\left(\mathbb{C}_{0}\right)$ is called outer if it maps $H^{2}\left(\mathbb{C}_{0}\right)$ by multiplication onto a dense subset in $H^{2}\left(\mathbb{C}_{0}\right)$, see Staffans $[29]$, Hoffman [12] or Foias et al. [7] for more general definitions. From Theorem 6 we have the plant transfer function $P(s)=C_{\Lambda}(s I-\mathcal{A})^{-1} \mathcal{B}_{1}$ and the disturbance transfer function $W(s)=C_{\Lambda}(s I-\mathcal{A})^{-1} \mathcal{B}_{2}$. In the $H^{\infty}$ controller design it is necessary to have the inner-outer factorization of $W(s)$ (see [8] and [7]). The inner-outer factorization of $P(s)$ is more involved, which we will address elsewhere. Generally speaking, $P(s)$ and $W(s)$ being irrational, it might be difficult to get their inner-outer factorization. Here we show that the strict properness of $P(s)$ and the inner-outer factorization of $W(s)$ are obtained directly from the structure of the system.

Theorem 7. (i) $P, W \in H^{\infty}\left(\mathbb{C}_{0}\right)$; (ii) $P(s)$ tends uniformly to zero as $|s|$ goes to infinity inside each half-plane $\Re e(s) \geq \delta \geq 0 ; \quad$ (iii) $W(s)=\exp \left(-\frac{l_{2} s}{m_{2}}\right) \cdot W_{O}(s)$, where $W_{O}(s)$ is outer.

Proof of Theorem 7. (i) follows from Theorems 5 and 4 and the application of [32]. (ii) Remark that $(s I-\mathcal{A})^{-1} \phi=\int_{0}^{\infty} \mathrm{e}^{-s t} \mathrm{e}^{t \mathcal{A}} \phi$ for all $\phi \in X$ and $\Re e(s)>\omega_{0}(\mathcal{A})$. Using the definition of $C_{\Lambda}$ and the Lebesgue's dominated convergence theorem we have

$$
C_{\Lambda}(s I-\mathcal{A})^{-1} \mathcal{B}_{1}=\int_{0}^{\infty} \mathrm{e}^{-s t} C_{\Lambda} \mathrm{e}^{t \mathcal{A}} \mathcal{B}_{1} \mathrm{~d} t, \quad \forall \Re e(s)>\omega_{0}(\mathcal{A})
$$

By the properties of well-posedness and exponential stability $C_{\Lambda} \mathrm{e}^{t \mathcal{A}} \mathcal{B}_{1}$ belongs to $L_{-\epsilon}^{2}\left(\mathbb{R}^{+}\right)$for some $\epsilon>0$ (cf. Curtain [6]). By means of the Paley-Wiener theorem (see p. 131 in [12]) $P(s-\epsilon)$ is in $H^{2}\left(\mathbb{C}_{0}\right)$. Each function (in particular, our $P(s-\epsilon)$ here) in $H^{2}\left(\mathbb{C}_{0}\right)$ tends uniformly to zero as $|s|$ goes to infinity inside any closed plane $\Re e(s) \geq \delta>0$ (see p. 125, Ref. [12]). (iii) Let $W_{O}(s)=W(s) \exp \left(\frac{l_{2} s}{m_{2}}\right)$. We prove that both $W_{O}$ and $W_{O}^{-1}$ are in $H^{\infty}\left(\mathbb{C}_{0}\right)$. Then the theorem is proved. 
We set

$$
\begin{aligned}
& M_{1}(s)=\left[\begin{array}{ll}
\frac{l_{1}\left(K_{2}+s\right)}{m_{2}} & \frac{l_{1} \sqrt{K_{1} K_{2}}}{m_{2}} \\
\frac{l_{1} \sqrt{K_{1} K_{2}}}{m_{1}} & -\frac{l_{1}\left(K_{1}+s\right)}{m_{1}}
\end{array}\right], \\
& M_{2}(s)=\left[\begin{array}{ll}
-\frac{\left(l_{2}-l_{1}\right)\left(K_{1}+s\right)}{m_{1}(1-\beta)} & -\frac{\left(l_{2}-l_{1}\right) \sqrt{K_{1} K_{2}}}{m_{1}(1-\beta)} \\
-\frac{\left(l_{2}-l_{1}\right) \sqrt{K_{1} K_{2}}}{m_{2}} & \frac{\left(l_{2}-l_{1}\right)\left(K_{2}+s\right)}{m_{2}}
\end{array}\right] .
\end{aligned}
$$

Taking the Laplace transform in (3.11) and re-arranging the components we obtain the following un-coupled differential systems:

$$
\begin{aligned}
& \frac{\partial}{\partial x}\left(\begin{array}{c}
\hat{\phi}_{1} \\
\hat{\phi}_{3}
\end{array}\right)=M_{1}(s)\left(\begin{array}{c}
\hat{\phi}_{1} \\
\hat{\phi}_{3}
\end{array}\right) \\
& \frac{\partial}{\partial x}\left(\begin{array}{c}
\hat{\phi}_{2} \\
\hat{\phi}_{4}
\end{array}\right)=M_{2}(s)\left(\begin{array}{c}
\hat{\phi}_{2} \\
\hat{\phi}_{4}
\end{array}\right)
\end{aligned}
$$

satisfying the boundary conditions:

$$
\left\{\begin{array}{l}
\left(\begin{array}{c}
\hat{\phi}_{2}(0) \\
\hat{\phi}_{4}(0)
\end{array}\right)=\left(\begin{array}{l}
0 \\
1
\end{array}\right) \hat{y}(s) \\
\left(\begin{array}{l}
\hat{\phi}_{1}(1) \\
\hat{\phi}_{3}(1)
\end{array}\right)=\varpi\left[\begin{array}{ll}
0 & 1 \\
1-\beta & 0
\end{array}\right]\left(\begin{array}{l}
\hat{\phi}_{2}(1) \\
\hat{\phi}_{4}(1)
\end{array}\right) \\
\hat{d}(s)=\varpi^{-1} \hat{\phi}_{1}(0) .
\end{array}\right.
$$

(Recall that $\varpi$ has been defined in (3.5).) The unique solution of (4.7) gives $W^{-1}(s)$ as follows:

$$
W^{-1}(s)=[1,0] \mathrm{e}^{M_{1}(s)}\left[\begin{array}{ll}
0 & 1 \\
1-\beta & 0
\end{array}\right] \mathrm{e}^{M_{2}(s)}\left[\begin{array}{l}
0 \\
1
\end{array}\right] .
$$

We set

$$
\begin{gathered}
\widetilde{M}_{1}(s)=\operatorname{diag}\left(0,-l_{1}\left(\frac{1}{m_{1}}+\frac{1}{m_{2}}\right) s\right), \\
\widetilde{M}_{2}(s)=\operatorname{diag}\left(-\left(l_{2}-l_{1}\right)\left(\frac{1}{m_{1}(1-\beta)}+\frac{1}{m_{2}}\right) s, 0\right) .
\end{gathered}
$$

We write

$$
\left\{\begin{array}{l}
M_{1}(s)-\left(d \frac{l_{1}}{m_{2}}\right) s=\widetilde{M}_{1}(s)+C_{1} \\
M_{2}(s)-\left(\frac{l_{2}-l_{1}}{m_{2}}\right) s=\widetilde{M}_{2}(s)+C_{2}
\end{array}\right.
$$


where $C_{1}$ and $C_{2}$ are constant matrices independent of $s$. It is easy to see that

$$
\left\|\exp \left(\widetilde{M}_{k}(s)+C_{k}\right)\right\|_{\mathcal{L}\left(\mathbb{R}^{2}\right)} \leq \exp \left(\left\|C_{k}\right\|_{\mathcal{L}\left(\mathbb{R}^{2}\right)}\right), \quad \forall k=1,2 .
$$

Using this inequality in (4.9) we prove that

$$
W_{O}^{-1}(\cdot)=W^{-1}(\cdot) \exp \left(\frac{-l_{2} \cdot}{m_{2}}\right) \in H^{\infty}\left(\mathbb{C}_{0}\right)
$$

Now it is sufficient to prove that $W_{O}(s)$ is analytic and bounded on some right half-plane because, from (i), $W_{O}(s)$ is analytic and bounded on any fixed stripe $0<\Re e(s) \leq \delta<\infty$. For this purpose we use again our idea in the proof of Theorem 6 . Using the same notation as there we can write

$$
W(s)=\left[\begin{array}{ll}
1 & 0
\end{array}\right](I-\widetilde{H}(s) K)^{-1} \widetilde{H}(s)\left[\begin{array}{l}
1 \\
0
\end{array}\right] .
$$

It is easy to see that $\exp \left(\frac{l_{2}}{m_{2}}\right) \widetilde{H} \in H^{\infty}\left(\mathbb{C}_{0}\right)$. By (4.4), W(s)exp $\left(\frac{l_{2} s}{m_{2}}\right)$ is analytic and bounded on some right half-plane.

Remark 6. The inner factor of $W(s)$ (which is a delay term) corresponds to the necessary time for the disturbance to arrive at the output in the heat exchanger network.

\section{Conclusions}

We have studied the dynamics of a heat exchanger network system in the frequency and time domain. This process is representative for a class of hyperbolic systems encountered in chemical engineering, such as heat exchangers, gas absorbers and irrigation canals. The paper shows how this system is transformed into a classical symmetric hyperbolic system, allowing us to prove exponential stability using the theorem of Rauch and Taylor. We have also proposed Lyapunov function candidates for proving the exponential stability of some other hyperbolic systems. The heat exchanger system has an unbounded control operator and an unbounded observation operator. We have shown that the system is regular and its transfer functions are in $H^{\infty}$. We have given the inner-outer factorization of some of them. Although the paper is a case study, the theory and the method that we have proposed in the paper are general and can be applied for a class of processes governed by symmetric hyperbolic systems. The results presented here are essential for various controller design methods ( cf. $H^{\infty}$ controllers [7, 8,10], optimal feedback controllers [29], output feedback controllers [6,31,33] and PI controllers $[17,18,22,37])$ to be applied for this class of processes.

\section{Appendix}

Computing $\xi_{\text {adj }}$ is equivalent to solving the following differential equation

$$
\left\{\begin{array}{l}
\frac{\partial g(x)}{\partial x}=A_{1}^{-1} B_{2} g(x)-A_{1}^{-1} f(x) \\
g_{3}(0)=g_{4}(0)=0 \\
{\left[\begin{array}{l}
g_{1}(1) \\
g_{2}(1)
\end{array}\right]=D_{\text {adj }}\left[\begin{array}{l}
g_{3}(1) \\
g_{4}(1)
\end{array}\right]} \\
\left\langle\xi_{\text {adj }}, f\right\rangle_{H}=\varrho \cdot g_{1}(0) .
\end{array}\right.
$$


We set $e_{1}=(1,0,0,0)$ and $N_{1}=A_{1}^{-1}$. Let $N_{2}$ be the $2 \times 4$ matrice such that $N_{2}=\left[I \mid D_{\text {adj }}\right]$. The differential equation has a unique solution $g \in \mathcal{D}\left(A^{*}\right)$ for each $f \in H$. By direct computation we find that

$$
g_{1}(0)=\varrho \cdot e_{1} \cdot\left\{\left[\begin{array}{l}
I \\
0
\end{array}\right]\left(N_{2} \mathrm{e}^{N_{1} \cdot B_{2}}\left[\begin{array}{l}
I \\
0
\end{array}\right]\right)^{-1} N_{2}-I\right\} \int_{0}^{1} \mathrm{e}^{N_{1} \cdot B_{2}(1-\tau)} N_{1} f(\tau) \mathrm{d} \tau
$$

Thus we obtain

$$
\left(\xi_{\text {adj }}(x)\right)^{*}=\varrho \cdot e_{1} \cdot\left\{\left[\begin{array}{l}
I \\
0
\end{array}\right]\left(N_{2} \mathrm{e}^{N_{1} \cdot B_{2}}\left[\begin{array}{l}
I \\
0
\end{array}\right]\right)^{-1} N_{2}-I\right\} \mathrm{e}^{N_{1} \cdot B_{2}(1-x)} N_{1} .
$$

Obviously $\xi_{\text {adj }}(x)$ is an analytic function of $x$.

This work has been done in part while the first author was visiting the Academy of Mathematics and System Sciences, Chinese Academy of Sciences, in 1999 and 2000, with the support of the joint research program PRA M97-05 FranceChina. He has benefited from his discussions with Professor D.X. Feng for a more general proof like that of Theorem 6. He would like to thank Dr. G. Weiss, Imperial College, London, for many references (e.g. $[17,18]$ and [33]) and corrections that he has done on our paper. The anonymous reviewers are also acknowledged for their constructive suggestions.

\section{REFERENCES}

[1] C.D. Benchimol, A note on weak stabilizability of contraction semigroups. SIAM J. Control Optim. 16 (1978) 373-379.

[2] H. Bounit, H. Hammouri and J. Sau, Regulation of an irrigation canal system through the semigroup approach, in Proc. of the International Workshop Regulation of Irrigation Canals: State of the Art of Research and Applications. Marocco (1997) 261-267.

[3] S.X. Chen, Introduction to partial differential equations. People Education Press (in Chinese) (1981).

[4] V.T. Chow, Open channel hydraulics. Mac-Graw·Hill Book Company, New York (1985).

[5] J.M. Coron, B. d'Andréa-Novel and G. Bastin, A Lyapunov approach to control irrigation canals modeled by Saint-Venant equations, in European Control Conference ECC'99. Karlsruhe (1999).

[6] R.F. Curtain, Equivalence of input-output stability and exponential stability for infinite-dimensional systems. Math. Systems Theory 21 (1988) 19-48.

[7] C. Foias, H. Özbay and A. Tannenbaum, Robust Control of Infinite Dimensional Systems. Frequency Domain Methods. Springer, Hong Kong, Lecture Notes in Control and Inform. Sci. 209 (1996).

[8] B.A. Francis and G. Zames, On $H^{\infty}$-optimal sensitivity theory for SISO feedback systems. IEEE Trans. Automat. Control 29 (1984) 9-16.

[9] J.C. Friedly, Dynamic Behavior of Processes. Prentice-Hall, Inc., Englewood Cliffs, New Jersey (1972).

[10] J.P. Gauthier and C.Z. Xu, $H^{\infty}$-control of a distributed parameter system with non-minimum phase. Int. J. Control 53 (1991) 45-79.

[11] K.M. Hangos, A.A. Alonso, J.D. Perkins and B.E. Ydstie, Thermodynamic approach to the structural stability of process plants. AIChE J. 45 (1999) 802-816.

[12] H. Hoffman, Banach Spaces of Analytic Functions. Prentice-Hall Inc., Englewood Cliffs (1962).

[13] F.L. Huang, Characteristic conditions for exponential stability of linear dynamical systems in Hilbert spaces. Ann. Differential Equations 1 (1985) 43-56.

[14] H.O. Kreiss, O.E. Ortiz and O.A. Reula, Stability of quasi-linear hyperbolic dissipative systems. J. Differential Equations 142 (1998) 78-96.

[15] P.D. Lax and R.S. Phillips, Local boundary conditions for dissipative symmetric linear differential operators. Comm. Pure Appl. Math. 13 (1960) 427-455.

[16] T.S. Li, Global Classical Solutions for Quasilinear Hyperbolic Systems, Research in Applied Mathematics, edited by P.G. Ciarlet and J.-L. Lions. John Willey \& Sons, New York (1994).

[17] H. Logemann, E.P. Ryan and S. Townley, Integral control of infinite-dimensional linear systems subject to input saturation. SIAM J. Control Optim. 36 (1998) 1940-1961.

[18] H. Logemann and S. Townley, Low gain control of uncertain regular linear systems. SIAM J. Control Optim. 35 (1997) $78-116$.

[19] K.A. Morris, Justification of input/output methods for systems with unbounded control and observation. IEEE Trans. Automat. Control 44 (1999) 81-85. 
[20] O.E. Ortiz, Stability of nonconservative hyperbolic systems and relativistic dissipative fluids. J. Math. Phys. 42 (2001) 1426-1442.

[21] A. Pazy, Semigroups of Linear Operators and Applications to Partial Differential Equations. Springer-Verlag, New York (1983).

[22] S.A. Pohjolainen, Robust multivariable PI-controllers for infinite dimensional systems. IEEE Trans. Automat. Control 27 (1985) 17-30.

[23] J. Prüss, On the spectrum of $C_{0}$-semigroups. Trans. Amer. Math. Soc. 284 (1984) 847-857.

[24] J. Rauch, Symmetric positive systems with boundary characteristic of constant multiplicity. Trans. Amer. Math. Soc. 291 (1985) 167-187.

[25] J. Rauch and M. Taylor, Exponential decay of solutions to hyperbolic equations in bounded domain. Indiana Univ. Math. J. 24 (1974) 79-86.

[26] R. Rebarber, Conditions for the equivalence of internal and external stability for distributed parameter systems. IEEE Trans. Automat. Control 38 (1993) 994-998.

[27] D.L. Russell, Controllability and stabilizability theory for linear partial differential equations: Recent progress and open questions. SIAM Rev. 20 (1978) 639-739.

[28] D. Salamon, Realization theory in Hilbert space. Math. Systems Theory 21 (1989) 147-164.

[29] O.J. Staffans, Feedback representations of critical controls for well-posed linear systems. Int. J. Robust Nonlinear Control 8 (1998) 1189-1217.

[30] G. Weiss, Admissible observation operators for linear semigroups. Israel J. Math. 65 (1989) 17-43.

[31] G. Weiss, Regular linear systems with feedback. Math. Control, Signals 83 Systems 7 (1994) 23-57.

$[32]$ G. Weiss, Transfer functions of regular linear systems. Part I: Characterizations of regularity. Trans. Amer. Math. Soc. 342 (1994) 827-854.

[33] G. Weiss and R.F. Curtain, Dynamic stabilization of regular linear systems. IEEE Trans. Automat. Control 42 (1997) 4-21.

[34] C.Z. Xu and D.X. Feng, Linearization method to stability analysis for nonlinear hyperbolic systems. C. R. Acad. Sci. Paris Sér. I Math. 332 (2001) 809-814.

[35] C.Z. Xu and J.P. Gauthier, Analyse et commande d'un échangeur thermique à contre-courant. RAIRO APII 25 (1991) $377-396$.

[36] C.Z. Xu, J.P. Gauthier and I. Kupka, Exponential stability of the heat exchanger equation, in Proc. of the European Control Conference. Groningen, The Netherlands (1993) 303-307.

[37] C.Z. Xu and H. Jerbi, A robust PI-controller for infinite dimensional systems. Int. J. Control 61 (1995) 33-45.

[38] C.Z. Xu, Exponential stability of a class of infinite dimensional time-varying linear systems, in Proc. of the International Conference on Control and Information. Hong Kong (1995).

[39] C.Z. Xu, Exact observability and exponential stability of infinite dimensional bilinear systems. Math. Control, Signals $\mathcal{E}$ Systems 9 (1996) 73-93.

[40] C.Z. Xu and G. Sallet, Proportional and Integral regulation of irrigation canal systems governed by the Saint-Venant equation, in 14th IFAC World Congress. Beijing, China (1999).

[41] C.Z. Xu and D.X. Feng, Symmetric hyperbolic systems and applications to exponential stability of heat exchangers and irrigation canals, in Proc. of the MTNS'2000. Perpignan (2000).

[42] B.E. Ydstie and A.A. Alonso, Process systems and passivity via the Clausius-Planck inequality. Systems Control Lett. 30 (1997) 253-264. 